\title{
The Use of Biologically-inspired Algorithms for the Optimization of Machining Parameters
}

\author{
Goran Miodragović ${ }^{*}$, Radovan Bulatović2 , Slobodan Ivanović ${ }^{1}$, Marina Bošković ${ }^{2}$ \\ ${ }^{1}$ College of Applied Professional Mechanical Studies, Trstenik (Serbia) \\ ${ }^{2}$ The Faculty of Mechanical and Civil Engineering in Kraljevo of the University in Kragujevac, Kraljevo (Serbia)
}

\begin{abstract}
This paper proposes the application of biologically -inspired algorithms to determine optimal parameters of metal cutting. The objective functions are operation time, cost per product, and surface roughness. Model of metal cutting is nonlinear, constrained problem. As an example of biologically -inspired algorithm for solving optimization problems, in this paper we have applied the Cuckoo search algorithm, and the Firefly algorithm. Contemporary analysis of these two methods, as well as their hybridization and the experimental results show that the use of biologically -inspired algorithms is applicable to parameter problems optimization of metal cutting.
\end{abstract}

Keywords: Cuckoo Search algorithm, Firefly algorithm, Hybridization, Machining parameters, Non-linear optimisation, Optimization

\section{INTRODUCTION}

Economical machining has always held an important place in determining the machining parameters. In practice, technologists and operator on the machine selected processing parameters on the basis of either their experience or using information from a database or from catalog of tools. However, there is rarely such values do often do guarantee the effectiveness of data processing or minimal processing costs. Processing costs minimization involved in how to determine includes the cutting speed, step and depth of cut, which will give optimum results.

When setting these parameters, special attention is given to the constraints that are characteristic for the chosen method of cutting, or that are determined by the machining method, machine tools, tools and workpiece. These constraints, among others, includes: a tool resistance; cutting force, required cutting power, the stability of the cutting zone, temperature field in the cutting zone, dimensional accuracy, surface quality, as well as the relationship between rough and finish machining.

There are a number of methods to deal with technoeconomic optimization of machining parameters. Some of these methods are concerned only with the machining in single pass. Parameters which have to be optimized, in this case, are the cutting speed and the feed rate. It is assumed that in this single pass, estimated depth of cut which is at the same time the maximum possible, what in most cases is not so. In other methods, the optimized parameters are either the number of passes (at a constant depth of cut) or the cutting depth per pass and cutting speed and feed rate. For this method of optimization (cost minimization or profit maximization), there are a variety of techniques, such as differential calculus, regression analysis, linear programming, geometric programming and stochastic, computer simulation.
Some of these methods do not consider previously mentioned constraints, because a large number of constraints complicates the problem of optimization of machining parameters using classical (deterministic) methods. Deterministic methods are used in cases where the objective function has no several local minima, there is no point where the gradient is not defined, or where the objective function is continuous. In situations where the objective function has many local minima, where there are a points where the gradient is not defined, and the objective function is discontinuous, heuristic methods such as genetic algorithms, simulated annealing, particle swarm optimization, Cuckoo Search algorithm, the Firefly Algorithm are used.

Heuristics means the way when we use trial and error to come to an acceptable solution. Metaheuristics algorithms represent a more advanced type of heuristic algorithms. These algorithms use a certain compromise between the random and local search. Metaheuristics algorithms have two important features: the intensification and diversification. Diversification means the search at the global level, while the intensification is based on the search at the local level. Algorithms are divided into the algorithms based on population and algorithms based on trajectory.

In this paper, we propose the use of hybrid algorithm, the firefly and cuckoo searches in optimizing the parameters of metal cutting.

\section{FIREFLY ALGORITHM}

Firefly algorithm was first developed by Xin-She Yang, 2007a. [1]. This algorithm is inspired by the behaviour and movement of fireflies in nature. Yang showed the superiority of this algorithm over the existing traditional optimization algorithms. Yang believes that each swarm can be associated with a Lévy flight. Thus, Lévy flight firefly algorithm is formulated. 


\subsection{The behaviour of fireflies}

There are about 2000 species of fireflies and they mainly inhabit temperate and tropical regions. Most of them produces a brief flash that creates a beautiful sight in the sky. Method of sowing is characteristic of each species. Basic functions of sowing are attracting partners (communication) and to attract potential victims. Also, seeding can be a warning sign. Females respond to a unique method of sowing the male within the same species. In some species the females can mimic the way planting other species in order to lure and ate males.

Planting firefly can be formulated as a method for the realization of the objective function optimization.

\subsection{Explanation of the algorithm}

Firefly algorithm can be considered as metaheuristics algorithm based on the swarm of fireflies and inspired by sieving. It is presented as an iterative procedure based on the numerical population. Factors interact with each other by means of sowing (natural light) which allows them to search the premises of the objective function. Appropriates it is a solution in which factor firefly glowing in proportion to its quality, in the considered problem. Each firefly attracts partners, regardless of sex, and thus build the search space, which is much more efficiently searched.

It is necessary to define two key things in this algorithm: first the changes in planting and second the attraction, [1].

In the simplest case, sifting fireflies, and at a certain location $\mathrm{x}$ can be written as:

$$
I(x) \propto f(x)
$$

The appeal of $\beta$ is relative and it varies with the distance $r_{i j}$, between firefly $i$ and $j$. In addition, the intensity decreases with seeding distance from the source, because the absorption is carried out in air. We can see that the attraction varies in proportion to the degree of absorption. The intensity of planting varies inversely proportional to the law:

$$
I(r)=I_{s} / r^{2}
$$

where $I_{s}$ is the intensity of the light source. The intensity of screening and $(r)$ varies with distance $r$ monotonically and exponentially. For a given environment with a fixed absorption coefficient of light will be:

$$
I=I_{0} e^{-\gamma r}
$$

where $I_{0}$ is the intensity of the actual planting. The value of $\gamma$ represents the change in attractiveness with increasing distance from the switch. The parameter $\gamma$ is an important part in determining the speed and convergence behaviour of the algorithm with the firefly. There are two limiting cases when $\gamma \rightarrow 0$ and $\gamma \rightarrow \infty$. When $\gamma \rightarrow 0$ the attraction has a constant value of $\beta=\beta_{0}$. In the case when $\gamma \rightarrow \infty$ attraction is close to zero, corresponding to a completely random search. In many embodiments typically ranges from 0.01 to 100 .

To avoid the singularity at $r=0$ in the expression using Gaussian $I_{s} / r^{2}$ formula:

$$
I=I_{0} e^{-\gamma r^{2}}
$$

The attractiveness of a firefly is proportional to the intensity of screening and can be defined by the equation:

$$
\beta=\beta_{0} \cdot e^{-\gamma r^{2}}
$$

where is $r$ the distance between two of the firefly and $\beta_{0}$ is their attraction when $r=0$. To calculate the exponential function takes longer than the computation rate $1 /\left(1+r^{2}\right)$. Therefore, if necessary, the previous relation can be approximated as:

$$
\beta=\frac{\beta_{0}}{1+\gamma r^{2}}
$$

The characteristic distance is based on the last two relations can be defined as $A=1 / \gamma$. Above this value the attraction is significantly changed.

The reality is attractive function $\beta(r)$ monotonically decreasing and can be represented as:

$$
\beta(r)=\beta_{0} e^{-\gamma r^{m}}
$$

For fixed $\gamma$ characteristic distance becomes:

$$
\Gamma=\gamma^{-1 / m} \rightarrow 1, m \rightarrow \infty
$$

The distance between two of the firefly $i$ and $j x_{i}$ and $x_{j}$, respectively, is defined as the distance Descartes:

$$
r_{i j}=\left\|x_{i}-x_{j}\right\|=\sqrt{\sum_{k=1}^{d}\left(x_{i, k}-x_{j, k}\right)^{2}}
$$

where $x_{i}, k$-th component of the spatial coordinates $x_{i}$ the $i$-th switch.

The movement of $i$-th firefly is attractive another more brilliant firefly $j$ and is defined as :

$$
x_{i}=x_{i}+\beta_{0} e^{-\gamma r_{i, j}^{2}}\left(x_{j}-x_{i}\right)+\alpha \varepsilon_{i}
$$

In equation (10) the second member is determined on the basis of attractiveness, while the third member is random (random) character. In the third article mentioning a vector random variable $\varepsilon_{i}$ which is taken from a Gaussian or uniform distribution.

In accordance with the above principles can be established optimization algorithm firefly, Figure 1. [1].

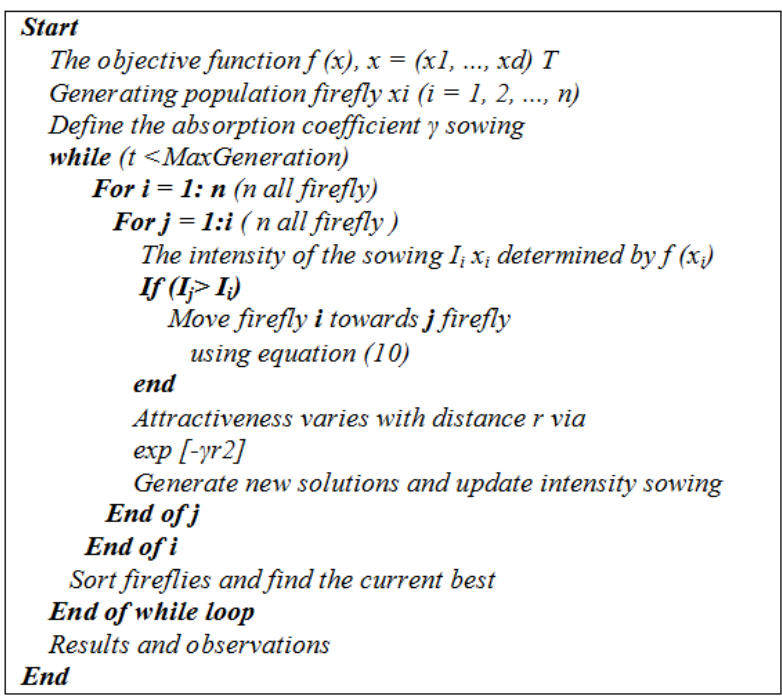

Figure 1. Pseudo code. Optimization firefly algorithm

\section{CUCKOO SEARCH ALGORITHM}

Cuckoo Search (CS) is also metaheuristics optimization algorithm, inspired by the biological behavior of a cuckoo searching for a nest where they can lay their eggs. This algorithm, as proposed by Yang and Deb. 


\subsection{The behavior of Cuckoo}

Cuckoo lay their eggs in the nests of other birds and bird hosts take care of the egg, and then chicken. Cuckoo usually chooses birds nest in which eggs are already set, so you can be sure that the first chicken hatched from an egg chicken. Some species are adapted to the cuckoo lay their eggs in the nests of other birds, so their eggs are very similar to the eggs of birds of the host. When hatched cuckoo chick, it instinctively pushed out of the nest eggs or chicks bird host in order to receive all the food from the new "parents". In addition, cuckoo can mimic the call of bird's species in whose nest is located. If, finally, the bird hosts realize that it is in their set are the cuckoo's nest, they either remove or abandon the nest.

\subsection{Explanation of the algorithm}

In this optimization algorithm, each nest represents a potential solution. The cuckoo reproduction process in the algorithm is simplified by three rules, [4]:

1. Each cuckoo lays an egg in a randomly chosen nest;

2. The best nests carry over to the next generation of cuckoos;

3. The number of available host nests is fixed (limited), and the egg laid by a cuckoo is discovered by the host bird with a probability pa, which ranges $[0,1]$. Birds can detect only the worst nests so that they are losing from the population.

CS has a simple algorithm, and its code is given in Yang and Deb (2010), [4]. The initial population of nests with the size $n$, which are randomly distributed over the search space, is generated first. The randomly chosen initial solutions of design variables are defined in the search space by the lower and upper boundaries.

The new nest, for example $i$-th, is generated according to the following law

$$
x_{i}^{(t+1)}=x_{i}^{(t)}+\alpha \otimes \operatorname{Lévy}(\lambda)
$$

where $\alpha>0$, is the step size whose value depends on the optimization problem, and $t$ is the current generation. Step size is multiplied by the random numbers with Lévy's distribution, and such random motion is called Lévy flight.

In this research work [4] a Levy flight in which the step-lengths are distributed according to the following probability distribution:

$$
L e^{\prime} v y u=t^{-\lambda} \quad 1<\lambda \leq 3 .
$$

In standard CS algorithm [4], parameters $p_{a}$ and $\alpha$ are very important in fine tuning of solution vector and appropriate selection of their values can result to the global solutions. However, values of these parameters are constant in the standard CS algorithm, Fig. 2.

Valian et al. (2013), [5], have introduced dynamic changes of these parameters in each generation, in solving complex engineering problem. If the value of probability $p_{a}$ is small, and the value of parameter $\alpha$, which represents step size, is large, such values can result in very slow convergency in CS algorithm. Otherwise, if the value of $p_{a}$ is large and the value of $\alpha$ is small, the speed of convergence is very fast and algorithm can not find the best solution.

The idea of ICS algorithm is that these parameters are adjustable in each generation, because in that way better solutions of algorithm can be achieved.

Begin
The objective function $f(x), x=\left(x_{1}, x_{2}, \ldots, x_{d}\right)^{T}$
Generate initial population of
$n$ host nest $x_{i}(i=1,2, \ldots, n)$
While $(t<$ MaxGeneration) or (stop Criterion)
Get a cuckoo randomly by Lévy flights
Evaluate its quality/fitness $F_{i}$
Choose a nest among $n$ (say. J) randomly
If $\left(F_{i}>F_{j}\right)$
$\quad$ Replace j by the new solution
End If
A fraction (pa) of whore nests are abandon
And a new ones are built
Keep the best solutions (or nests with quality solutions)
Rank the solutions and find the current best
End while
Figure 2. Pseudo code. Optimization Cuckoo search
algorithm
SEARCH AND FIREFLY)

Hybridizing algorithm means are combination of analyzed optimization algorithms (Cuckoo search and Firefly algorithm).

In this paper, the proposed algorithm is obtained so that the Cuckoo search algorithm implements part of Firefly algorithm. In the classical Cuckoo search algorithm, Fig 2, when is reached the probability of finding the worst nest $\left(p_{a}\right)$, it nest is leaving and these nest is assigned a new value for a random distribution. In the proposed algorithm, instead of "abandoning" the worst nests, reducing the probability of finding the worst nests installing firefly algorithm, Fig. 3. In other words, "bad nest" is replaced with the best firefly.

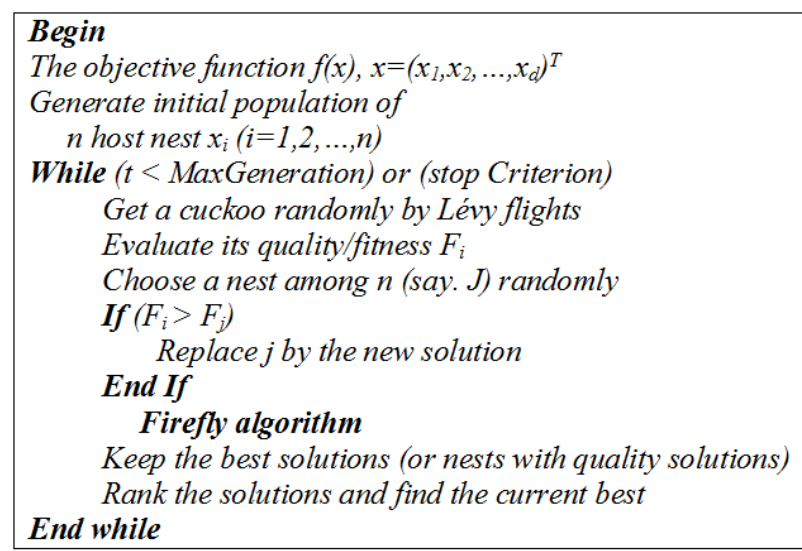

Figure 3. Pseudo code. Hybrid Optimization Cuckoo search \& FireFly algorithm

\section{OPTIMIZATION MODEL}

The main objectives in the optimization of machining processes are reducing processing costs, increasing productivity and profits. Of course, it is possible to combine these goals and then approaches to solving optimization problems with multiple objectives. By accessing the optimization problem, it is necessary to acquire the necessary knowledge about the process. To set the optimization model it is necessary to define: the objective function, the function of the process conditions, 
functions and limitations of optimization criteria. In machining processes, the most common functions of the state are: force (resistance) cutting, cutting power, cutting temperature, tool wear, tool life and surface quality. As the objective function: processing time, processing costs, processing accuracy, productivity, cost, profit are usually taken Restrictions relating to performance of machine tools, tools and work piece are set as function limitations [10]. The criteria of optimization are usually: minimization of time and processing costs or maximizing productivity and profits or maximum surface quality, Fig. 4.

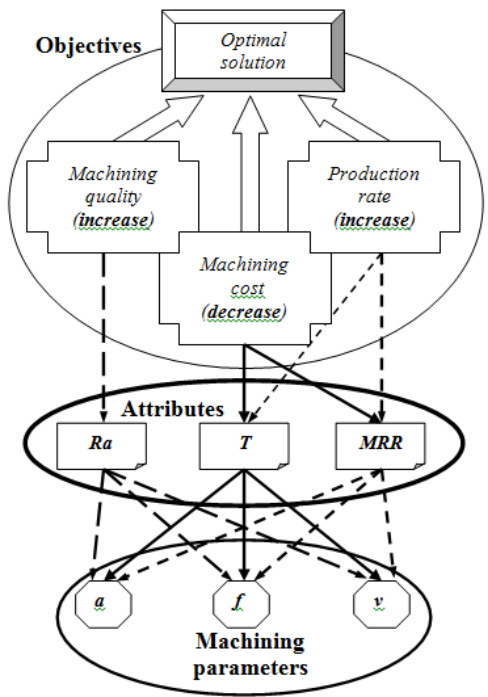

Figure 4. Representation structure of the objectives, attributes and cutting parameters

Without going into the details of generating optimization models, as a base for verification of the proposed hybrid optimization algorithm, a model that aims to reduce costs and processing time is presented and raise productivity.

The parameters that can be optimize are cutting speed (v), feed rate (a) and the cutting depth (a), [10].

\subsection{Operation time}

Operation time is measured as the entire time necessary for the manufacture of a product $\left(T_{P}\right)$. It is the function of the material removal rate $(M R R)$ and of the tool life $(T)$ [10]:

$$
T_{P}=T_{S}+V_{R M} \cdot \frac{1+\frac{T_{C}}{T}}{M R R}+T_{i}
$$

where is $T_{s}$ the tool set $u$ time, $T_{C}$ the tool change time, $T_{i}$ idle time between two consecutive cuts, $T$ tool life and $V_{R M}$ the volume of the removed material. In some cases the $T_{s}, T_{C}, T_{i}$ and $V_{R M}$ are constants so that $T$ is the function of $M R R$ and $T$.

The material removal rate can be calculated using the following equation [10]:

$$
M R R=1000 \cdot v \cdot f \cdot a
$$
cutting depth.

where is $v$ the cutting speed, $f$ feeding rate and $a$

The tool life is measured as the average time between the tool changes or tool sharpenings. The relation between the tool life and the parameters is expressed with the well known Taylor's formula [10]:

$$
T=\frac{k_{T}}{v^{a_{1}} \cdot f^{a_{2}} \cdot a^{a_{3}}}
$$

where $k_{T}, a_{1}, a_{2}$ and $a_{3}$, are constants relevant to a specific tool-workpiece combination [10].

\subsection{Operation cost}

The operation cost $\left(C_{P}\right)$ can be expressed as the cost per product, as follows [10]:

$$
C_{P}=T_{P} \cdot\left(\frac{C_{t}}{T}+C_{l}+C_{0}\right)
$$

where is $T_{P}$ necessary time for the manufacture of a product, $T$ tool life, $C_{t}$ the tool cost, $C_{l}$ the labour cost and $C_{0}$ overhead cost.

\subsection{Cutting quality}

The most important criterion for the determination of the surface quality is roughness [10]:

$$
R_{a}=k \cdot v^{k_{1}} \cdot f^{k_{2}} \cdot a^{k_{3}}
$$

where $k_{1}, k_{2}, k_{3}$, and $k$ are constants relevant to a specific tool-workpiece combination.

\subsection{Constrains}

Space solutions to be scanned in the search for the optimum conditions when routing is limited by technological and practical requirements relating to the installed power of machines $(P)$, the maximum cutting force $(F)$, the available range of steps $\left(f_{\min }-f_{\max }\right)$ and cutting speeds $\left(v_{\min }-v_{\max }\right)$ on the machine and the maximum, $a_{\max }$, and minimum, $a_{\min }$, cut depth.

$$
P=\frac{F \cdot v}{6122 \cdot \eta}
$$

where $F$ is the cutting force, $v$ cutting speed , $\eta$ efficiencies.

As we mentioned feed rate and speed must match the range of the selected machine and cutting depth can not exceed a given range:

$$
\begin{aligned}
& f_{\text {min }} \leq f \leq f_{\text {max }} \\
& v_{\text {min }} \leq v \leq v_{\text {max }} \\
& a_{\text {min }} \leq a \leq a_{\text {max }}
\end{aligned}
$$

\subsection{The objective function}

The task here is to find the optimum cutting conditions involving the same three decision variables: speed (v), feed rate (f), and cut depth (a). The objectives are operation time, cost per product, and surface roughness, which all must be minimized for a better machining operation. In the case of many incomparable and contradictory objectives the ideal solutions satisfying all requirements are very rare. In order to ensure the evaluation of mutual influences and the effects between the objectives it is recommendable to determine the multiattribute function of the manufacturer $(F)$ representing the company's/ manufacturer's overall preference. The following manufacturer's implicit value function $[10,13]$ is selected:

$$
\begin{aligned}
F & =0.42 \cdot e^{-0.22 \cdot T_{P}}+0.36 \cdot e^{-0.32 \cdot C_{P}}+0.17 \cdot e^{-0.26 \cdot R_{a}} \\
& +\frac{0.05}{1+1.22 \cdot T_{P} \cdot C_{P} \cdot R_{a}}
\end{aligned}
$$




\section{EXPERIMENTAL RESULTS}

The machining operation considered here is a turning process of machining a cast steel blank using NC lathe with a HSS cutting tool. The multi-objective formulation is given below $[10,13]$ :

\{following three objective functions

Minimize $F_{1}(x)=T_{P}(x)$

Minimize $F_{2}(x)=C_{P}(x)$

Minimize $F_{3}(x)=R_{a}(x)$

are replaced with single objective function $\}$

Minimize $F\left(T_{P}, C_{P}, R_{a}\right)$ from Eq. (25)

subject to $g_{1}(x)=1-\frac{P(x)}{P_{\max }} \geq 0$

$g_{2}(x)=1-\frac{F(x)}{F_{\text {max }}} \geq 0$

$70 \leq v \leq 90(\mathrm{~m} / \mathrm{min})$

$0.1 \leq f \leq 2(\mathrm{~mm} / \mathrm{rev})$

$0.1 \leq a \leq 0.5(\mathrm{~mm})$

$P_{\text {max }}=5 \mathrm{~kW}$

$F_{\text {max }}=230 \mathrm{~N}$

where:

$$
\begin{aligned}
& T_{P}(x)=0.12+2313.76 \cdot\left(\frac{\left.1+\frac{0.26}{T(x)}\right)}{M R R(x)}\right)+0.04 \\
& C_{P}(x)=\left(\frac{13.55}{T(x)}+0.39\right) \cdot T_{P}(x) \\
& R_{a}(x)=1.001 \cdot\left(v^{0.0088} \cdot f^{0.3232} \cdot a^{0.3144}\right) \\
& T(x)=1575134.21 \cdot\left(v^{-1.7} \cdot f^{-1.55} \cdot a^{-1.22}\right) \\
& M R R(x)=1000 \cdot v \cdot f \cdot a \\
& F(x)=1.38 \cdot f^{1.18} \cdot a^{1.26} \\
& P(x)=0.000626 \cdot v \cdot f^{0.24} \cdot a^{0.11} \\
& \quad x=\left[x_{1} x_{2} x_{3}\right]=[v f a]
\end{aligned}
$$

\subsection{Cucko search algorithm}

Algorithm parameters that are used to solve this problem are:

- $\quad$ The number of chest is $n=280$

- The value of probability is $p_{a}=0,75$

\begin{tabular}{|c|c|}
\hline Project variables & The values \\
\hline$x_{1}=v$ & $77.2232[\mathrm{~m} / \mathrm{min}]$ \\
\hline$x_{2}=f$ & $1.8704[\mathrm{~mm} / \mathrm{rev}]$ \\
\hline$x_{3}=a$ & $4.6863[\mathrm{~mm}]$ \\
\hline Objective function $\boldsymbol{F}$ & 0,9007 \\
\hline \multicolumn{2}{|l|}{ Constraints } \\
\hline$g_{1}$ & 0,9909 \\
\hline$g_{2}$ & 0,9922 \\
\hline
\end{tabular}

- The number of iteration is $N=500$.

The parameters and the results obtained in solving the optimization problem are shown in the Table 1.The values of the particular objective function for which a minimum was searched are given in Table 2.

Table 1. Values of objective function, project variables and constraints for cutting parameters optimization
Table 2. Values of objective functions

\begin{tabular}{|r|r|}
\hline Objective function & \multicolumn{1}{c|}{ The values } \\
\hline$T_{P}$ & $0.1634[\mathrm{~min}]$ \\
\hline$C_{P}$ & $0.1033[€ /$ piece] \\
\hline$R_{a}$ & $2,0694[\mu \mathrm{m}]$ \\
\hline
\end{tabular}

6.2. Firefly algorithm

Algorithm parameters that are used to solve this problem are:

- $\quad$ The number of fireflies is $n=40$

- $\quad$ The step size is $\alpha=2.5$

\begin{tabular}{|c|c|}
\hline Project variables & The values \\
\hline$x_{1}=v$ & $70.9737[\mathrm{~m} / \mathrm{min}]$ \\
\hline$x_{2}=f$ & $0,6558[\mathrm{~mm} / \mathrm{rev}]$ \\
\hline$x_{3}=a$ & $2.1327[\mathrm{~mm}]$ \\
\hline Objective function $\boldsymbol{F}$ & 0,9271 \\
\hline \multicolumn{2}{|l|}{ Constraints } \\
\hline$g_{1}$ & 0,9939 \\
\hline$g_{2}$ & 0,9984 \\
\hline
\end{tabular}

- The attraction is $\beta=0.2$

- $\quad$ The parameter $\gamma$ from equation (3) is $\gamma=1$

Table 3 and Table 4 show the results obtained from Firefly optimization algorithm. As noted, the obtained results are slightly worse than results which we obtained from Cuckoo search algorithm.

Table 3. Values of objective functions

Table 4: Values of objective functions

\begin{tabular}{|r|r|}
\hline Objective function & \multicolumn{1}{c|}{ The values } \\
\hline$T_{P}$ & $0.1833[\mathrm{~min}]$ \\
\hline$C_{P}$ & $0.0744[€ /$ piece] \\
\hline$R_{a}$ & $1.1056[\mu \mathrm{m}]$ \\
\hline
\end{tabular}

6.3. The hybrid algorithm

\begin{tabular}{|c|c|}
\hline Project variables & The values \\
\hline$x_{1}=v$ & $90[\mathrm{~m} / \mathrm{min}]$ \\
\hline$x_{2}=f$ & $2[\mathrm{~mm} / \mathrm{rev}]$ \\
\hline$x_{3}=a$ & $5[\mathrm{~mm}]$ \\
\hline Objective function $\boldsymbol{F}$ & 0,8929 \\
\hline \multicolumn{2}{|l|}{ Constraints } \\
\hline$g_{1}$ & 0,9938 \\
\hline$g_{2}$ & 0,9984 \\
\hline
\end{tabular}

The results of optimization received by using hybridized algorithm, given in Table 5. and Table 6., are the better than result obtained with Cuckoo search algorithm and Firefly Algorithm. This is because instead of rejecting "bad" nests, the part of Firefly algorithm which refer to attract fireflies is used.

Table 5. Values of objective functions

Table 6. Values of objective functions

\begin{tabular}{|r|r|}
\hline Objective function & \multicolumn{1}{c|}{ The values } \\
\hline$T_{P}$ & $0.1626[\mathrm{~min}]$ \\
\hline$C_{P}$ & $0.1247[€ /$ piece] \\
\hline$R_{a}$ & $2,1611[\mu \mathrm{m}]$ \\
\hline
\end{tabular}

Results graphical representation of the objective function $F$ is shown in Fig. 5, respectively, presentation the individual objective function TP, CP and $\mathrm{R}_{\mathrm{a}}$ is shown in Fig. 6. 


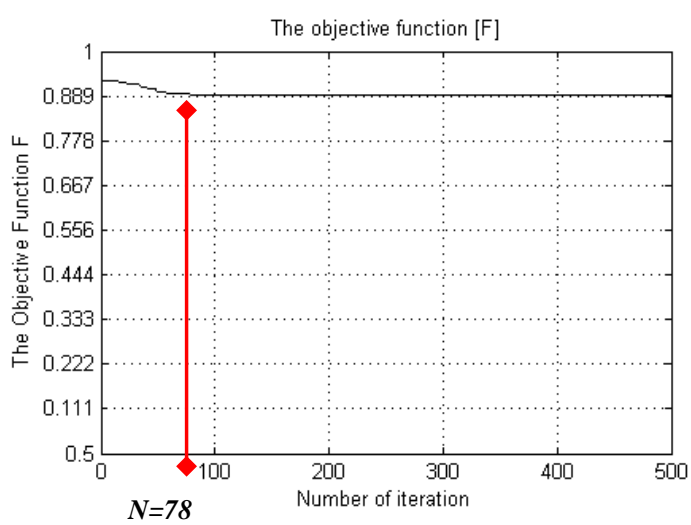

Figure 5.The Objective function $F\left(T_{P}, C_{P}, R_{a}\right)$

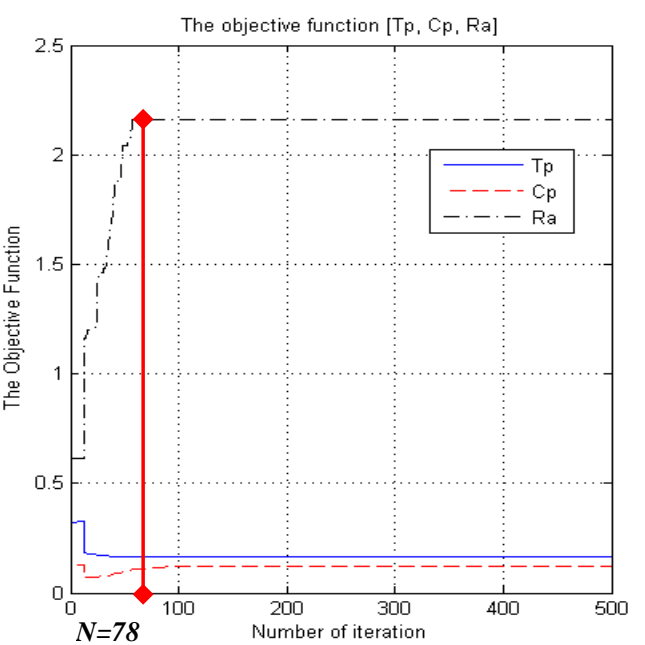

Figure 6.The Objective functions:

$T P(v, f, a), C P(v, f, a), R a(v, f, a)$

From Fig. 5. and 6. it can be noticed that after 78 iterative passages algorithm reached optimal solution. The speed of convergence depends of the selected parameter $\left(p_{a}\right)$, so that its variation may slow or speed up the convergence.

\section{CONCLUSION}

This paper describes a biologically - inspired algorithms for the optimization of cutting parameters. It is also shown attempt of hybridization of these two algorithms: Cuckoo search and Firefly algorithm. The obtained results showed that hybrid Cuckoo \& Firefly algorithm gives the better result, than Cuckoo search and Firefly algorithm.

This paper also showed that hybridization is possible, but it is necessary to be caution when combining optimization algorithms, because in addition to a satisfactory solution, small dispersion of received results is needed during the iterative process.

The future researches and development of hybrid algorithm should go towards achieving better and optimal results with shortening the search time. In this sense, it is necessary to do some modifications of the algorithm and the improvement of existing code.

\section{REFERENCES}

[1] Yang XS, "Firefly Algorithms for Multimodal Optimization”, Stochastic Algorithms: Foundations and Applications, SAGA 2009, vol. 5792, pp. 169178 (2009).

[2] Yang XS, "Engineering optimization: an intoduction with metaheuristic applications”, John Wiley \& Sons, (2010).

[3] R. Bulatović, G. Bošković, M. Savković, M. Gašić, "Improved Cuckoo Search (ICS) algorthm for constrained optimization problems”, Latin American Journal of Solids and Stuctures, 2014, vol. 11, no. 8, pp. 1349-1362, (2014).

[4] Yang, X.S., Deb, S., "Engineering Optimisation by Cuckoo Search”, International Journal of Mathematical Modelling and Numerical Optimisation, vol 2(4), 330-343., (2010).

[5] Valian, E., Tavakoli, S., Mohanna, S., Haghi, A., "Improved cuckoo search for reliability optimization problems”, Computers \& Industrial Engineering, vol. 64, 1, pp. 459-468, (2013).

[6] Talbi E, "Metaheuristics: from design to implementation.”, Hoboken, New Jersey: John Wiley \& Sons, (2009)

[7] Gandomi AH, Yang XS, Alavi H.A. "Cuckoo search algorithm: a metaheuristic approach to solve structural optimization problems”, Engin with comput, 2013, vol. 29, pp. 17-35

[8] Saibal K. Pal, C.S Rai i Amrit Pal Singh, "Comparative study of Firefly Algorithm and Particle Swarm Optimization for Noisy Non-Linear Optimization Problems”, I.J.Intelligent Systems and Applications

[9] Miodragović G, Bošković M, "The use of the firefly algorithm for solving problems of applied mechanics”. IMK-14 - Istraživanje i razvoj, vol. 19, pp. 56-61, (2013)

[10] D. Kalyanmoy, D. Rituparna, "Hybrid Evolutionary Multi-Objective Optimization of Machining Parameters”, Engineering Optimization, vol 44, Issue 6, pp. 685-706,2012.

[11] F. Cus and J. Balic, "Optimization of cutting process by GA approach”, Robotics and ComputerIntegrated Manufacturing, vol. 19(1-2), pp.113-121, 2003.

[12] B. Malakooti, J. Wang, E.C. Tandler, "A sensorbased accelerated approach formulti-attribute machinability and tool life evaluation", International Journal of Production Research, vol. 28, pp. 23732392, (1990).

[13] U. Zuperl, F. Cus, “Optimization of Cutting Conditions During Machining by Using Neural Networks", International Conference on Flexible Automation and Intelligent Manufacturing 2002, Dresden, German, (2002). 